\title{
Multi-objective Optimization Approach to Power Allocation in Multibeam Systems
}

CONFERENCE PAPER · SEPTEMBER 2012

DOI: $10.2514 / 6.2012-15202$

CITATION

1

6 AUTHORS, INCLUDING:

Alexis Aravanis

National Technical University of Athens

6 PUBLICATIONS 3 CITATIONS

SEE PROFILE

\section{Grégoire Danoy}

University of Luxembourg

72 PUBLICATIONS 200 CITATIONS

SEE PROFILE
READS

42
Bhavani Shankar Mysore

University of Luxembourg

42 PUBLICATIONS 101 CITATIONS

SEE PROFILE

Björn Ottersten

University of Luxembourg

556 PUBLICATIONS $\quad 10,553$ CITATIONS

SEE PROFILE 


\title{
Multi-objective Optimization Approach to Power Allocation in Multibeam Systems
}

\author{
Alexios Aravanis ${ }^{1}$ \\ National Technical University of Athens, 15780 Zografou, Greece \\ University of Luxembourg, L-1359Kirchberg, Luxembourg \\ Bhavani Shankar M. $\mathrm{R}^{2}$ and Gregoire Danoy ${ }^{3}$ \\ University of Luxembourg, L-1359 Kirchberg, Luxembourg \\ Pantelis-Daniel Arapoglou ${ }^{4}$ \\ National Technical University of Athens, 15780 Zografou, Greece \\ Panayottis G. Cottis ${ }^{5}$ \\ National Technical University of Athens, 15780 Zografou, Greece \\ and \\ Bjorn Ottersten ${ }^{6}$ \\ University of Luxembourg, L-1359 Kirchberg, Luxembourg
}

\begin{abstract}
Current satellite systems employ multi-beam technology that allows for the dynamic allocation of the system resources (i.e. bandwidth and power), based on changing traffic distribution to provide substantial capacity gains. Often such an allocation tends to utilize the maximum DC power available, whereas a minimization of the same is desirable. This paper deals with the power allocation with respect to two seemingly conflicting objectives, namely the maximization of the available system throughput and the minimization of the system power consumption. A multi-objective
\end{abstract}

\footnotetext{
Student, School of Electrical and Computer Engineering, Iroon Polytechniou 9, non-member.

Research Associate, Interdisciplinary Centre for Security Reliability and Trust (SnT), Campus Kirchberg, 6, Rue Richard-Coudenhove Kalergi, non-member.

3 Research Associate, Computer Science and Communications Research Unit (CSC), Campus Kirchberg, 6, Rue Richard-Coudenhove Kalergi, non-member.

4 Research Associate, School of Electrical and Computer Engineering, Division of Information Transmission Systems and Material Technology, Iroon Polytechniou 9, non-member.

5 Professor, School of Electrical and Computer Engineering, Division of Information Transmission Systems and Material Technology, Iroon Polytechniou 9, non-member.

6 Director, Interdisciplinary Centre for Security Reliability and Trust (SnT), Campus Kirchberg, 6, Rue Richard-Coudenhove Kalergi, non-member.
} 
optimization approach is proposed for power allocation to handle the aforementioned objectives. A state-of-the-art multi-objective genetic algorithm (MOGA), i.e. the non-dominated sorting genetic algorithm II (NSGA-II), is used as optimization approach. The set of obtained solutions is presented in the form of a Pareto front, which provides complete information to the user concerning the trade-off between the traffic demand and power requirements.

\begin{tabular}{|c|c|c|}
\hline & & Nomenclature \\
\hline$N$ & $=$ & number of beams \\
\hline$N_{c}$ & $=$ & number of carriers \\
\hline$B_{T O T}$ & $=$ & total bandwidth \\
\hline$P_{T O T}$ & $=$ & total power \\
\hline$P_{b}$ & $=$ & beam power \\
\hline$P_{b, c}$ & $=$ & carrier power \\
\hline$B$ & $=$ & carrier bandwidth \\
\hline$P_{b, \text { con }}$ & $=$ & beam power constraint \\
\hline$R_{b, \text { req }}$ & $=$ & bit rate requested by user in beam b \\
\hline$R_{b, \text { off }}$ & $=$ & offered cumulative bit rate of all $\mathrm{N}_{\mathrm{c}}$ carriers in beam $\mathrm{b}$ \\
\hline
\end{tabular}

\section{Introduction}

$\boldsymbol{T}$-HE advent of new applications, including fixed and mobile multimedia interactive services, necessitate that satellite systems become more flexible. Design flexibility is essential to ensure that satellite systems can deal with emerging traffic requirements and unexpected market changes or the change in orbital location during the lifespan of the spacecraft. Flexibility could be viewed in four aspects with regard to coverage, power, frequency planning and higher layer functionalities (e.g. routing, switching) ${ }^{1}$. In exploiting these degrees of flexibility, multibeam satellites can play a key role since their design can easily be extended to support reconfigurability of power and frequency plans as well as routing and switching functionalities. This feature of multibeam systems along with the recent advances in Multi-Port Amplifier (MPA) and flexible Traveling Wave Tube Amplifier (TWTA) technology allow for efficient beam power allocation in response to the spatiotemporal variations of traffic demand.

In some previous work, optimization routine to efficiently apportion power across the beams to match the traffic demand has been considered. ${ }^{5}$ Such an optimization is considered to be subject to a maximum RF power constraint, which, in turn, assumes a maximum DC power consumption. Since DC power consumption is a critical factor, the possibility of minimizing DC power is also warranted. However efforts toward modifying the DC/ RF power consumption have not been explored in detail in literature. Therefore, the present paper explores the power allocation problem with respect to both seemingly conflicting objectives: maximization of the available system throughput and minimization of the system power consumption, subject to the aforementioned constraints.

In order to handle these objectives, the appropriate cost (or objective) functions to quantify the performance of each objective have to be selected. In the case of the system power consumption this function is just the overall system power consumption, but in the case of the available system throughput four different cost functions are found in literature, namely the differential and unmet system capacities ${ }^{7}$, satisfaction factor ${ }^{8}$ and aggregate fitness. For the identification of the most suitable cost function a preliminary study, a comparison of the four functions is conducted. Following the results of Ref. 5, where a systematic study of the appropriate optimization techniques for the maximization of the available throughput is performed, a Genetic Algorithm (GA) ${ }^{3}$ is adopted in the preliminary study. The results of the four cost functions employing the GA are then presented and compared and the best performing cost function is selected. Then, in order to enhance the system utilization, this cost function is used together with 
the minimization of the overall system power consumption as cost functions of a multi-objective optimization problem (MOP).

A MOP deals with the optimization of multiple conflicting objective functions. In such MOPs the order relation between solutions relies on the concept of dominance. A solution dominates another if it is strictly better in one objective and better or equal with regard to all other objectives. The optimal solution then, is not a single solution like in single-objective optimization problems, but a set of non-dominated solutions defined as Pareto optimal solutions. This set of solutions represents the compromise between the conflicting objectives and allow for the selection of the best solution, according to the preferences of the decision maker and the priority of each objective.

Subsequently a state-of-the-art multi-objective genetic algorithm (MOGA), the non-dominated sorting genetic algorithm II (NSGA-II) ${ }^{3}$ is employed for the resolution of this MOP. The set of solutions obtained is then presented in the form of a Pareto front (i.e. the image of the Pareto solutions in the objective space), providing complete information concerning the trade-off between the traffic demand and power requirements. The results are then analysed, demonstrating the potential benefits that arise from the use of the multi-objective approach.

The remainder of this paper is organized as follows. Section 2 presents the formulation of the resource optimization problem for multibeam architecture as a MOP. In the same section the optimization techniques applied to the problem in previous studies are presented and compared with the approach proposed in the present paper. Then the considered optimization techniques (GA for the preliminary study and NSGA-II for the multi-objective optimization) are described in section 3 . The preliminary study and the experimental results using the proposed multi-objective approach are presented and analysed in section 4 . Finally section 5 contains our conclusions and perspectives.

\section{Resource Optimization in Multibeam Architecture}

\section{Multibeam Architecture and System Resources}

Assume a multibeam satellite system with $\mathrm{b}=1, \ldots, \mathrm{N}$ beams, employing a typical four colour reuse pattern ( for e.g., two colours in frequency and two in polarization ), where the available total downlink bandwidth of the system $\mathrm{B}_{\text {Tот }}$ is equally distributed among the four colours. It is further assumed that the available bandwidth reused over the different colours is equally divided into the beams of each colour and each of these beams accommodates four carriers. A carrier represents the elementary system entity for conveying different streams of information and the bandwidth of each beam is equally divided among its carriers. The described layout could be visualized in Fig. 1, where the beam layout adopted for this paper's simulations is depicted.

Having allocated $\mathrm{B}_{\text {тот }}$ equally among the carriers, the resource allocation pertains to the appropriate allocation of the total available system power $\mathrm{P}_{\text {Tот. }} \mathrm{P}_{\text {TOт }}$ is a function of the platform total DC power on board the satellite, and must be allocated appropriately to each beam, so that the offered beam bit rate meets the user requirements in the same beam. The power allocated to each beam $\mathrm{P}_{\mathrm{b}}$, will then be equally divided among the $\mathrm{N}_{\mathrm{c}}$ carriers of the beam $\mathrm{P}_{\mathrm{b}, \mathrm{c}}=\mathrm{P}_{\mathrm{b}} / \mathrm{N}_{\mathrm{c}}$. Furthermore minimizing the overall power consumption while allocating the available $\mathrm{P}_{\mathrm{TOT}}$ is also warranted.

\section{B. Resource Optimization}

Following this analysis the resource optimization problem is formulated as a MOP. The target of this optimization is to determine the beam power $\mathrm{P}_{\mathrm{b}}$, for which:

1. The system capacity is maximized (cost function $\mathrm{f}_{1 \text { ) }}$.

2. The overall power consumption is minimized (cost function $\mathrm{f}_{2}$ ).

Subject to:

$>$ The power constrains of the system

$>$ The power constrains of each beam

For the system capacity maximization the following four candidate cost functions have been employed in past studies: 


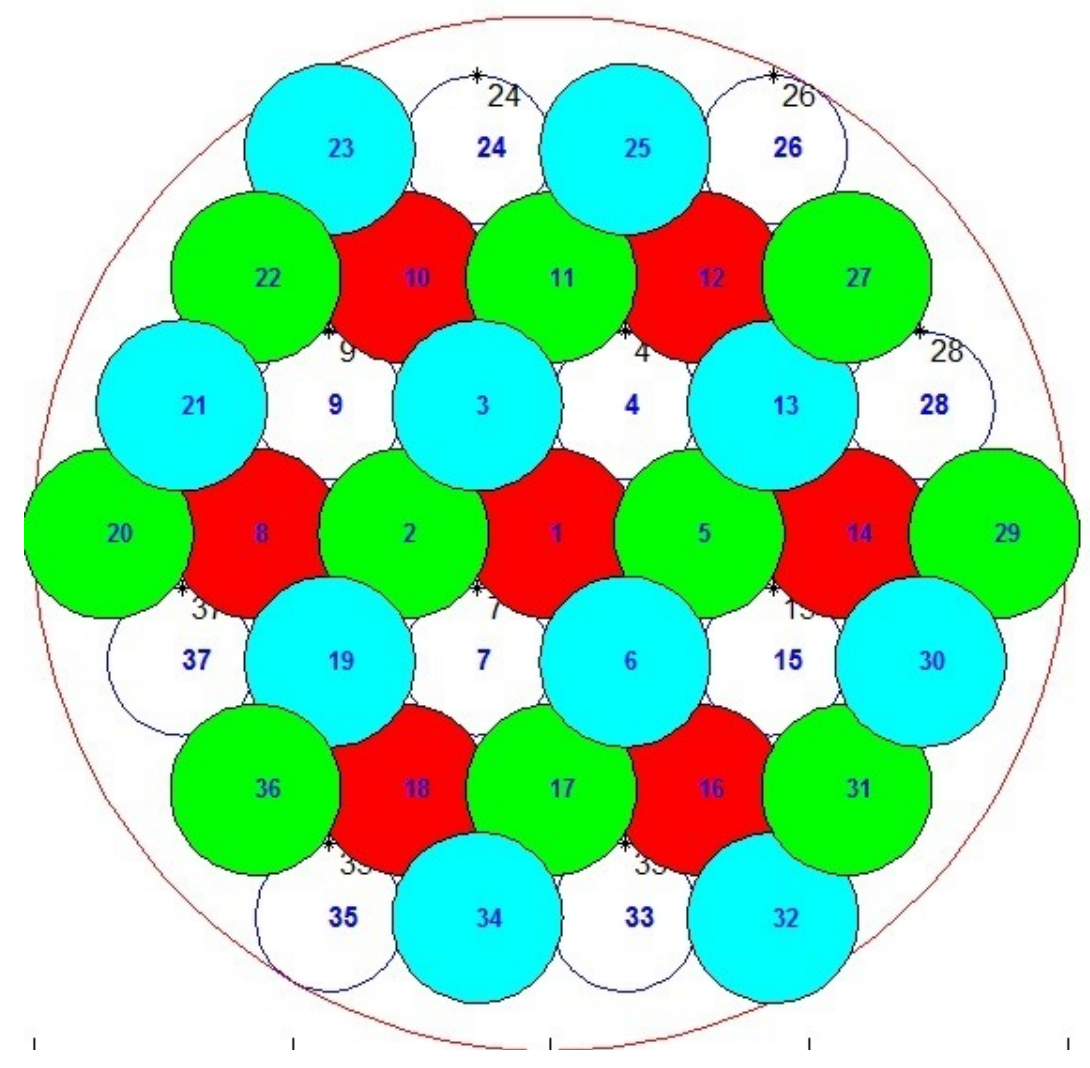

Figure. 1 Four colour reuse pattern, for a 37 circular beam layout

Differential System Capacity (DSC)

$$
\operatorname{Min} f_{1}=\sum_{\mathrm{b}=1}^{\mathrm{N}} \operatorname{abs}\left[\mathrm{R}_{\mathrm{b}, \text { req }}-\mathrm{R}_{\mathrm{b}, \text { off }}\right]
$$

According to Eq. (1), offered beam capacities exceeding or being less than the required beam capacities, both contribute to the figure of merit and drive the optimization closer to the requirements.

\section{Unmet System Capacity (USC)}

$$
\operatorname{Min} f_{1}=\sum_{\mathrm{b}=1}^{\mathrm{N}} \max \left[\mathrm{R}_{\mathrm{b}, \text { req }}-\mathrm{R}_{\mathrm{b}, \text { off }}, 0\right] \text {, }
$$

In Eq. (2), offered beam capacities exceeding the required do not contribute to the figure of merit and do not affect the optimization.

Satisfaction Factor (SF)

$$
\operatorname{Max} f_{1}=\frac{\sum_{\mathrm{b}=1}^{\mathrm{N}} \min \left[\mathrm{R}_{\mathrm{b}, \text { req }}, \mathrm{R}_{\mathrm{b}, \text { off }}\right]}{\sum_{\mathrm{b}=1}^{\mathrm{N}} \mathrm{R}_{\mathrm{b}, \text { req }}}
$$

The offered beam capacities exceeding the required capacity do not contribute to the figure of merit defined in Eq. (3). But in this case the recessive beam capacities are scaled by the cumulative required capacity.

Aggregate Fitness (AF)

$$
\operatorname{Max}_{1}=\sum_{b=1}^{\mathrm{N}} \frac{1}{1+\mathrm{abs}\left[\mathrm{R}_{\mathrm{b}, \text { req }}-\mathrm{R}_{\mathrm{b}, \text { off }}\right]}
$$


According to Eq. (4), offered beam capacities exceeding or being less than the required beam capacities, both contribute to the figure of merit, like in the case of the DSC, but these results are scaled down on a beam bases and the optimization is driven according to the sum of the individual beam fitness.

For the power consumption minimization the cost function employed is the following:

$$
\operatorname{Min} f_{2}=\sum_{\mathrm{b}=1}^{\mathrm{N}}\left[\mathrm{P}_{\mathrm{b}}\right]
$$

And the optimization is subject to the following constrains:

System power constraint

$$
\sum_{b=1}^{N}\left[P_{b}\right] \leq P_{T O T}
$$

Beam power constraints:

$$
\mathrm{P}_{\mathrm{b}} \leq \mathrm{P}_{\mathrm{b}, \text { con }}
$$

The offered, bit rate $R_{\mathrm{b}, \text { off }}$ is calculated as follows:

$$
\mathrm{R}_{\mathrm{b}, \mathrm{off}}=\sum_{\mathrm{c}=1}^{\mathrm{N}_{\mathrm{c}}} \mathrm{B} \times \mathrm{f}_{\mathrm{DVB}-\mathrm{S} 2}\left(\mathrm{SNIR}_{\mathrm{c}}\right)
$$

The function $\mathrm{f}_{\mathrm{DVB}-\mathrm{S} 2}\left(\mathrm{SNIR}_{\mathrm{C}}\right)$ quantifies the spectral efficiency of the various modulation and coding schemes employed by DVB-S2 as a function of the $\mathrm{SNIR}_{\mathrm{c}}$ of carrier c.

\section{SNIR Calculation}

The $\mathrm{SNIR}_{\mathrm{c}}$ of each carrier c, transmitted with power $\mathrm{P}_{\mathrm{b}, \mathrm{c}}$ and bandwidth $\mathrm{B}$ is calculated as follows ${ }^{4}$ :

$$
\mathrm{SNIR}_{\mathrm{c}}=\frac{\mathrm{a}_{\mathrm{b}}^{2} \mathrm{P}_{\mathrm{b}, \mathrm{c}}(\mathrm{OBO})}{\mathrm{N}_{0}\left(\mathrm{a}_{\mathrm{b}}\right) \mathrm{B}+\sum_{\mathrm{q} \in \Phi} \alpha_{\mathrm{q}}^{2} \mathrm{P}_{\mathrm{q}, \mathrm{c}}(\mathrm{OBO})+\mathrm{I}_{\mathrm{adj}}(\mathrm{B}, \mathrm{XPD})+\mathrm{I}_{\mathrm{adj}_{\mathrm{sat}}}+\mathrm{I}_{\text {inter }}\left(\mathrm{OBO}, \mathrm{C}_{\mathrm{b}}, \mathrm{Mod}\right)}
$$

$\Phi$ : is the set of co-channel beams in the coverage area with active carriers overlapping with the bandwidth of the intended carrier c (co-channel interference into intended beam b)

$a_{q}$ : is a gain factor encompassing the effect of: satellite antenna beam $q$, terminal receive antenna gain, free space loss, clear sky attenuation and rain attenuation

$\mathrm{N}_{0}$ : is the noise power spectral density which is a function of $\mathrm{a}_{b}$ because of the increase in noise temperature under rain fading conditions

$\mathrm{I}_{\text {adj_ch }}$ : accounts for adjacent channel interference due to filter imperfections (function of B), including spillover from the beams in orthogonal polarization if both polarizations are employed (function of XPD)

$\mathrm{I}_{\text {adj_sat }}$ : Inter-system interference caused by adjacent satellites

$\mathrm{I}_{\text {inter }}$ : Intermodulation interference

The notation $\mathrm{P}_{\mathrm{b}, \mathrm{c}}(\mathrm{OBO})$ denotes the dependence on the appropriate OBO according to the modulation scheme employed

\section{Single-objective Transformation of the Considered MOP}

An early approach in the direction of modifying the DC/RF power consumption along with the available system throughput is proposed in Ref. 6 . In this approach a number of cost functions to quantify the satisfaction of the traffic demand are proposed. These cost functions include a DC consumption parameter, in order to take the power consumption into account, while maximizing the available throughput of the system. This technique known as scalar approach is transforming a MOP into a single-objective problem, by combining different objective functions (i.e. the traffic demand satisfaction and the DC consumption minimization) into a single-objective function in a linear way.

This type of approach however, requires a priori knowledge on the considered problem, in order to find the appropriate weights for the linear representation of the different objective functions. That is because the Pareto optimal solution is the node of the objective space hyperplane, defined by the weight vector and the feasible space of the problem. Thus the Pareto border has to be known in order to select the corresponding weights to obtain the desirable optimal solution. 
A common misconception, is that the weights selected for the linearization of the MOP are representative of the relative importance of the different objectives. In these cases the objective space hyperplane defined by these weights, could intersect randomly with the feasible space of the problem, providing undesirable solutions.

Alternatively multiple weights can be used, that will be optimized in parallel along with the problem. However the computational cost of this process is significant and even this process cannot guarantee to provide all Pareto optimal solutions, since these are limited to the convex hull of the Pareto front (i.e. solutions in concave regions of the Pareto front cannot be found).

Furthermore the provided optimal solution of these scalar approaches is a single solution unlike the set of Pareto solutions, provided by the dominance based approaches, depriving the decision maker of the alternative solutions, suppressing useful information regarding the system's objective space.

The considered problem however is not known a priori. Hence, the present paper explores the power allocation problem as a strictly multi-objective problem (MOP) and not as a linear function of the various objectives. Formulation of the problem in a dominance based multi-objective setting seems to be novel in literature and in this course a state-of-the-art multi-objective genetic algorithm (MOGA), the nondominated sorting genetic algorithm II (NSGA-II) ${ }^{3}$,presented next, is employed for its resolution.

\section{Optimization Techniques}

\section{A. Genetic Algorithm (GA)}

The GA adopted in the preliminary study of the paper, is a population based metaheuristic, where the search for a "good" solution starts from an initial population of acceptable solutions. This technique is based on the Darwinian evolutionary model, which consists in the following iterative process. Two parents are first selected from the whole population with a given selection criterion. Then two genetic operators are sequentially applied with some probability, namely recombination (or crossover) that exchanges portions of the parents solution vectors, and mutation that randomly modifies the individual. Finally the generated offsprings are evaluated and inserted back into the population following a given criterion.

\section{B. Non-dominated Sorting Genetic Algorithm II (NSGA II)}

NSGA-II is the most popular and referenced dominance based multi-objective algorithm in literature. It is a GA with a non-structured population, that is used to obtain the new population after applying the typical genetic operators (i.e. selection, crossover, mutation). As a GA belongs to the metaheuristic techniques, and since metaheuristics do not guarantee the optimality of the obtained solution the goal of this technique is to provide an approximation of the Pareto optimal set with two necessary properties: convergence to the Pareto optimal solutions and uniform diversity (i.e. a good distribution of the obtained solutions around the Pareto optimal front).

In this course, all individuals of the initial population are ranked with respect to both convergence and diversity. All non-dominated solutions are assigned to the rank 1 and are subsequently removed from the population in order to assign the current non-dominated solutions to the rank 2 . This process is iterated for all individuals and then individuals within the same rank are ranked according to the distance from their surrounding individuals. Individuals with higher distances (i.e. good distribution) are ranked higher than others.

The individuals are then sorted according to their rank and the typical genetic operators are applied iteratively.

\section{Experimental Results}

For the simulations presented herein, the 37-beam system described in Fig. 1 has been simulated, where the antenna pattern is approximated by employing the Bessel function and the link budget is calculated assuming one user per beam, located at the beam edge (worst case position). The parameters of the link budget, are presented in Table 1. 
Table 1 Link Budget Parameters

\begin{tabular}{|l|c|}
\hline \multicolumn{2}{|c|}{ Link Budget Parameters For Capacity Results } \\
\hline Parameter & Value \\
\hline Frequency Band & Ku \\
\hline User Link Bandwidth $\mathbf{B}_{\mathbf{u}}$ & $46.875 \mathrm{MHz}$ \\
\hline HPA saturation Power $\mathbf{P}_{\boldsymbol{\tau}}$ & $80 \mathrm{~W}$ \\
\hline Max satellite antenna gain $\mathbf{G}_{\mathbf{T}}$ & $52 \mathrm{dBi}$ \\
\hline Output Back Off $\mathbf{O B O}$ & $5 \mathrm{~dB}$ \\
\hline Satellite EIRP & $66 \mathrm{dBW}$ \\
\hline Free Space Loss $\mathbf{L}$ & $212 \mathrm{~dB}$ \\
\hline Terminal Antenna Gain $\mathbf{G}_{\mathbf{R}}$ & $41.7 \mathrm{dBW}$ \\
\hline Terminal noise Temperature $\mathbf{T}$ & $207 \mathrm{~K}$ \\
\hline Receive $\mathbf{C} / \mathbf{N}$ & $20.2 \mathrm{~dB}$ \\
\hline External $(\mathbf{C} / \mathbf{I})_{\text {ExT }}$ & $30.0 \mathrm{~dB}$ \\
\hline
\end{tabular}

\section{A. Problem Instance Setup}

Simulating the above system, the cost functions defined in Section 2 are compared, based on their performance concerning the maximization of the available system throughput. The technique employed for the comparison of the cost functions is the most appropriate technique out of the standard metaheuristics techniques compared in Ref. 5, namely the GA. The solutions are encoded as 37-dimensional vectors, where each dimension represents the $\mathrm{R} / \mathrm{F}$ power of the respective beam. The optimization parameters selected for the simulations are shown in Table. 2.

Table 2. GA Optimization Parameters

\begin{tabular}{|l|c|}
\hline \multicolumn{1}{|c|}{ Parameter } & Value \\
\hline Population Initialisation & Random \\
\hline Population Size & 5856 \\
\hline Crossover Function & Uniform \\
Crossover Rate & 0.95 \\
\hline Mutation Function & Uniform \\
Mutation Rate & 0.05 \\
\hline Selection Function & Tournament \\
\hline Elite Individuals & 30 \\
\hline Fitness Evaluations & 234240 \\
\hline
\end{tabular}

\section{B. Single Objective Results (Preliminary Study)}

The performance assessment of the different cost functions is shown in Table. 3 . The results reported involve averaging over 15 independent runs, as the computational time needed for 234240 evaluations did not allow the use of a bigger sample. The statistical confidence in the comparisons is assessed by performing the Wilcoxon test ${ }^{2}$, with standard confidence level (0.95).

Table 3. Cost Function Performance Assessment

\begin{tabular}{|c|c|c|c|c|}
\hline \multirow{2}{*}{$\begin{array}{c}\text { Cost } \\
\text { Function }\end{array}$} & \multicolumn{3}{|c|}{ Capacity [Gbps] } & Power [W] \\
\cline { 2 - 5 } & Required & Offered & Unmet & \\
\cline { 2 - 5 } & 24.155 & 23.588 & 0.801 & 1758.2 \\
\hline DSC & 24.155 & 25.605 & 0.746 & 2145.7 \\
\hline USC & 24.155 & 23.946 & 0.748 & 2010.4 \\
\hline SF & 24.155 & 23.547 & 0.842 & 1752.5 \\
\hline AF & \multicolumn{4}{|c|}{} \\
\hline
\end{tabular}




\begin{tabular}{|c|c|c|c|c|}
\hline & \multicolumn{4}{|c|}{ (Overall Best over 15 runs) } \\
\hline DSC & 24.155 & 23.593 & 0.778 & 1743.9 \\
\hline USC & 24.155 & 24.879 & 0.709 & 2030.3 \\
\hline SF & 24.155 & 24.879 & 0.709 & 2030.3 \\
\hline AF & 24.155 & 23.588 & 0.802 & 1777.9 \\
\hline
\end{tabular}

The numerical results presented above demonstrate the suitability of the USC as a cost function, since the USC provides the lower unmet capacity of all. This result is not surprising since USC focuses on the minimization of the unmet capacity, ignoring the impact of the exceeding beam capacities on the optimization. Therefore the performance of the SF, which also ignores the impact of the exceeding beam capacities is similar, with that of USC, obtaining also the same overall best solution. The performance of the best USC result in the satisfaction of the traffic demand is depicted in Fig. 2.

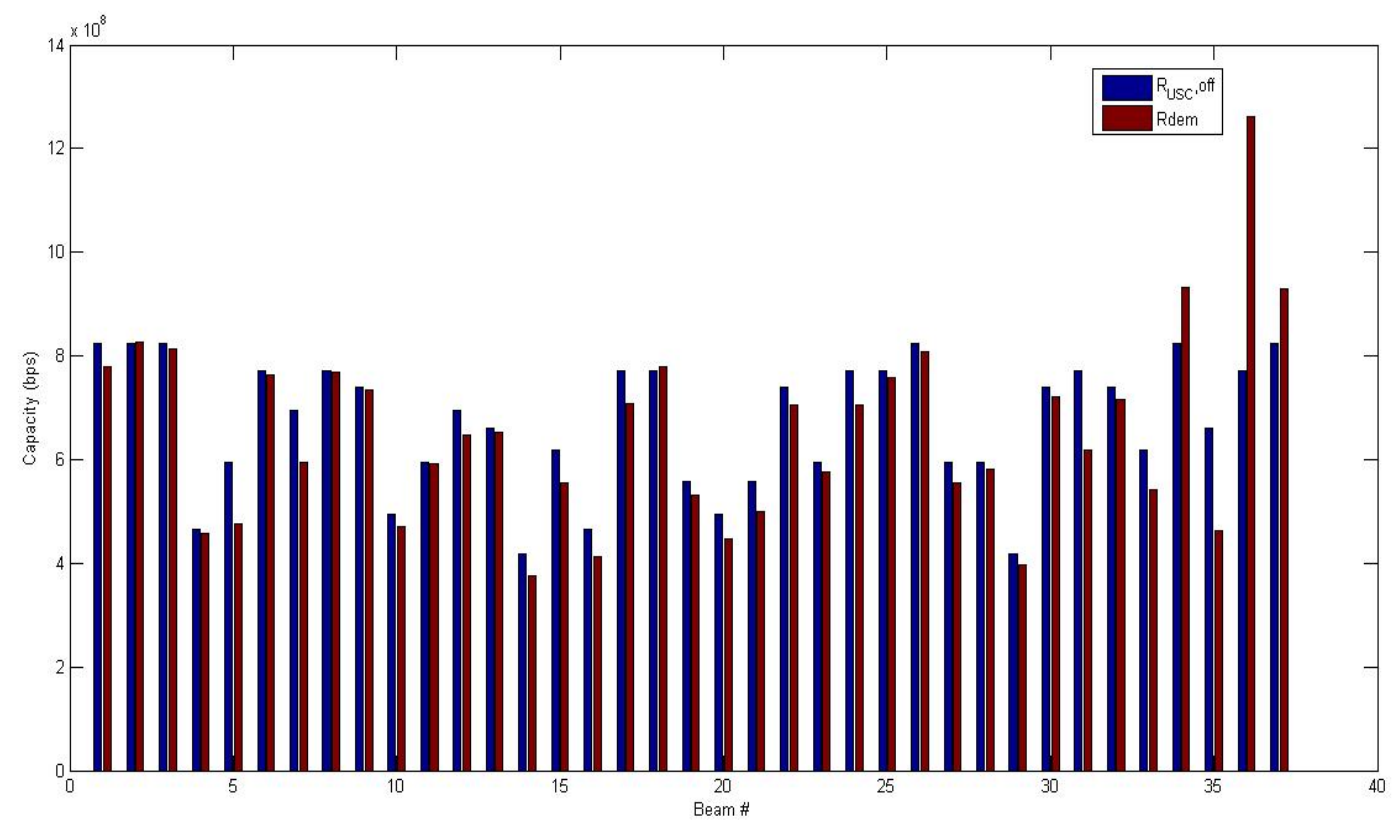

Figure.2 Single objective optimization performance (USC)

\section{Multi-objective optimization results}

The result presented in Fig. 2 is the best result provided for the maximization of the system throughput. This can be used as initial seed for the multi-objective approach ensuring that the result providing the best performance in terms of the capacity maximization will be included in the Pareto optimal set of solutions and possibly will be further enhanced.

Subsequently the obtained solution is used with the same encoding described above as an individual of the initial population of the NSGA-II. The target of the optimization employing the NSGA-II is to determine the power $\mathrm{P}_{\mathrm{b}}$ for which the Eq. (2) and (5), are minimized. The results reported from this optimization involve averaging of 15 independent runs and the optimization parameters selected are shown in Table. 4.

Table.4 NSGA-II Optimization Parameters

\begin{tabular}{|l|c|}
\hline \multicolumn{1}{|c|}{ Parameter } & Value \\
\hline Population Initialisation & Random \\
\hline Population Size & 6000 \\
\hline Crossover Function & Uniform \\
Crossover Rate & 0.9 \\
\hline
\end{tabular}




\begin{tabular}{|l|c|}
\hline Mutation Function & Uniform \\
Mutation Rate & 0.05 \\
\hline Selection Function & Tournament \\
\hline Fitness Evaluations & 240000 \\
\hline
\end{tabular}

The Pareto front obtained from the 15 runs is depicted in Fig. 3 providing complete information concerning the trade-off between offered capacity and power requirements, whereas the initial population provided by the single-objective approach is further enhanced.

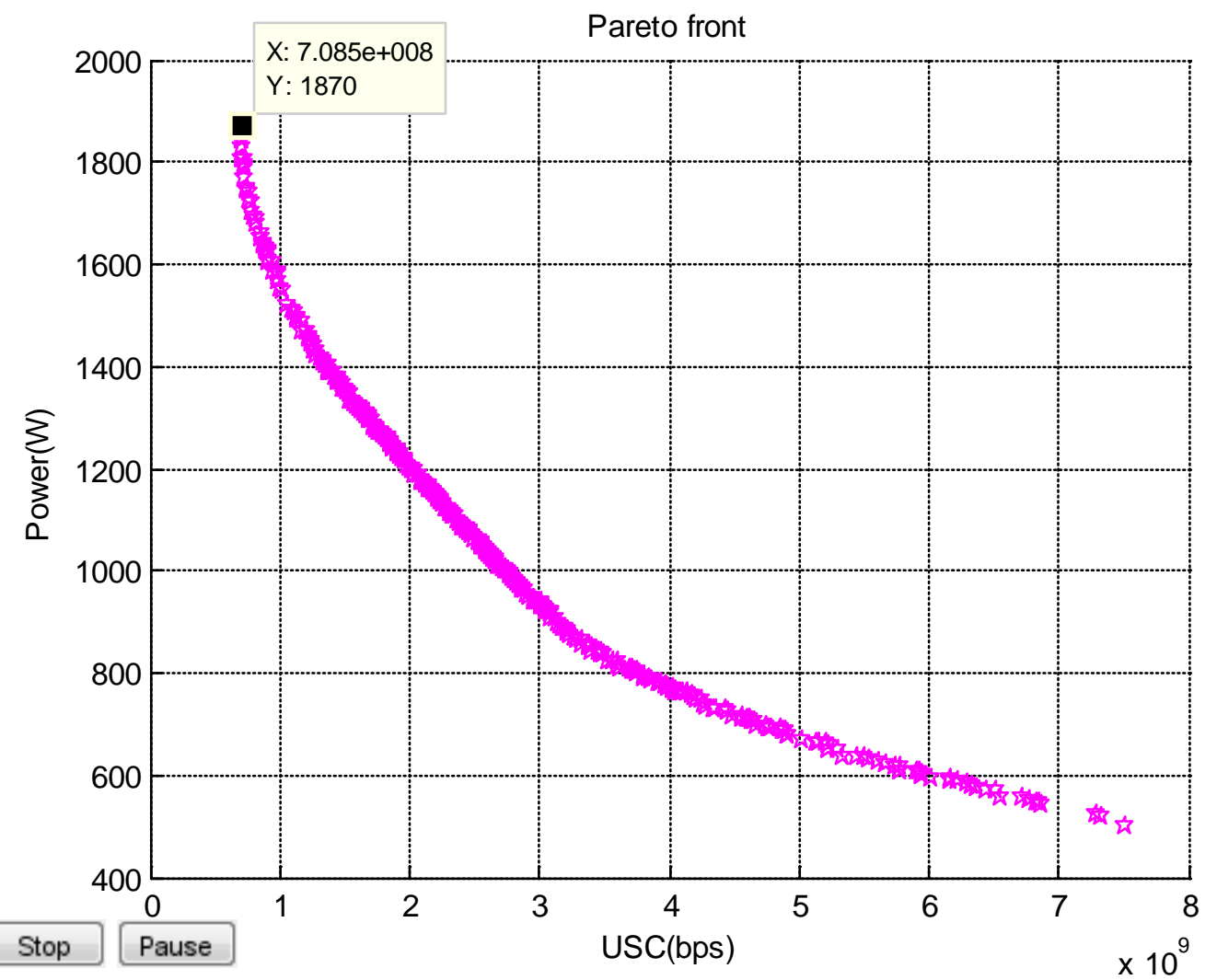

Figure. 3. Pareto front encompassing the enhanced result of the single-objective optimization.

From the above figure, it appears that the obtained Pareto front provides a large number of nondominated solutions as well as a very good diversity. It is also evident that the result of the single-objective optimization, shown in Table 3 has improved significantly in terms of Power. In particular the allocation obtained by the single-objective approach, consuming 2030.3W for an USC of 0.709Gbps dropped to a power consumption of $1870 \mathrm{~W}$ for the same USC. In other words the multi-objective approach allows for the system to perform the same in terms of satisfying the traffic demand while saving 7,9\% power.

Furthermore the slope of the left part of the Pareto front is steep enough, to allow for even more significant power savings, in case the user is willing to let the USC deteriorate by little. Fig. 4 shows the left part of the Pareto front, demonstrating the trade-off between the satisfaction of the traffic demand and the power requirements. In this case the user can save up to $12 \%$ power by decreasing the USC by $2.7 \%$. It is evident that the Pareto front obtained provides complete information regarding the trade-off between the two objectives, enabling the decision maker (in this point of view, the satellite operator) to adjust to the emerging traffic requirements according to the priority of the two objectives. 


\section{Conclusion}

The present paper demonstrates how current multibeam satellite systems can benefit from the use of multi-objective approaches for the allocation of their resources, which is novel approach in literature. The interesting aspect is that the two objectives are seemingly conflicting: maximization of the available system throughput and the minimization of the system power consumption due to their relation to the power and yet the proposed technique can improve one objective by 7,9\%, without deteriorating the other. Moreover complete information to the satellite operator regarding the trade-off of the different objectives is provided, enabling a full exploitation of the available flexibility of the system yielding significant capacity gains and power efficiency, which results to the prolongation of the spacecraft's life expectancy.

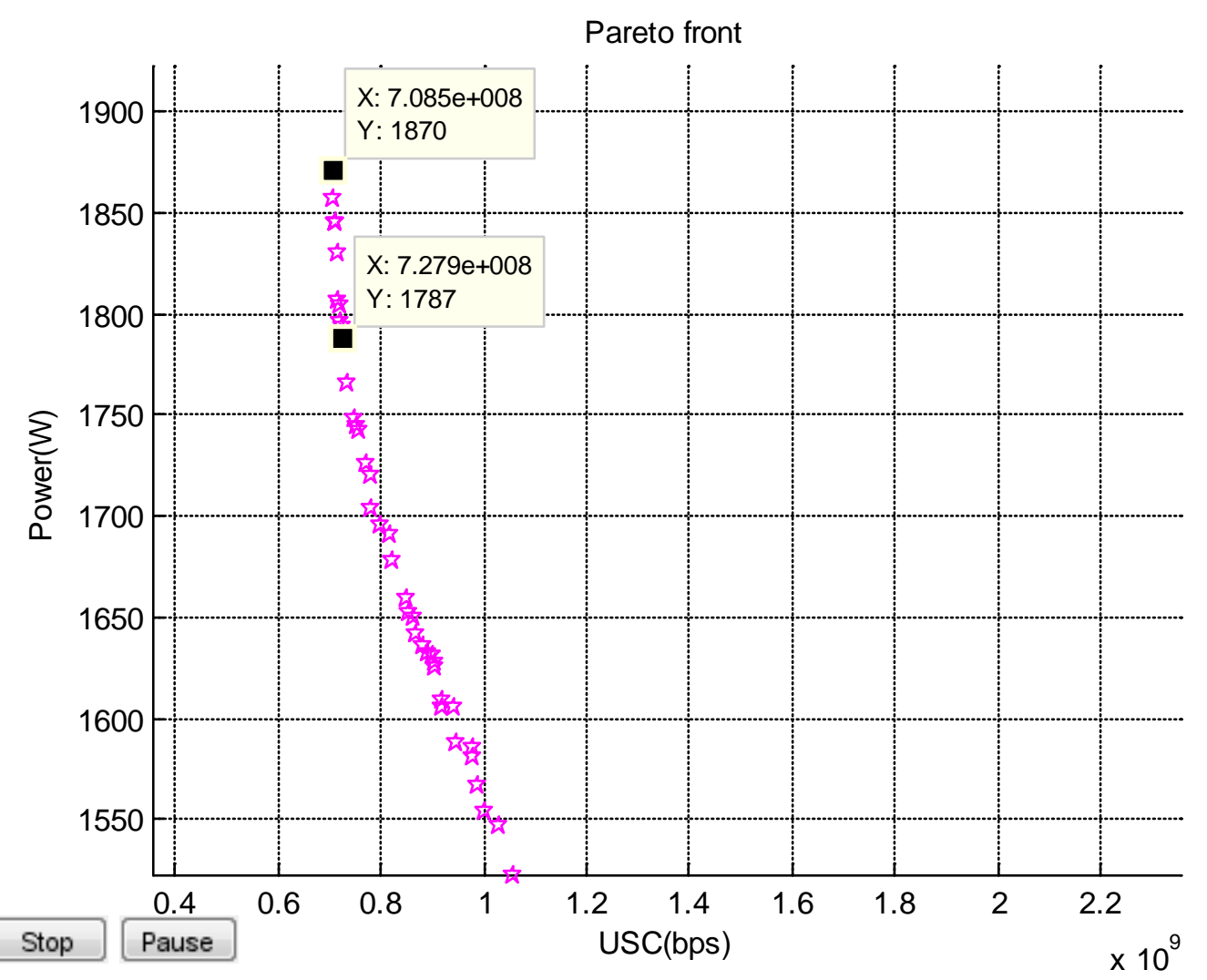

Figure.4 Pareto front, zoom in

\section{References}

${ }^{1}$ Balty C., Gayrard J-D., Agnieray P., “Communication Satellites to enter new age of flexibility,” Acta Astronautica, Vol. 65, Apr. 2009, pp. 75-81.

${ }^{2}$ Wilcoxon F., “Individual comparisons by ranking methods,” Biometrics Bulletin Vol. 1, No.6, 1945, pp. 80-83.

${ }^{3}$ Talbi E.G., Metaheuristics from design to implementation, John Wiley \& Sons, Inc., New Jersey, 2009.

${ }^{4}$ Bousquet M., Maral G., Satellite Communications Systems, John Wiley \& Sons, Inc., United Kingdom, 2009.

${ }^{5}$ Aravanis A., Shankar B., Danoy G., Arapoglou D., Cottis P., Ottersten B., "Power allocation in Multibeam satellites - A hybrid-Genetic Algorithm approach,” 2nd ESA Workshop on Advanced Flexible Telecom Payloads, 17-19 April 2012. 
${ }^{6}$ Gabellini P., D’Agristina L., Alagha N., Angeletti P., “On the Cost Function for the Performance Optimization of Multi-Beam Broadband Satellite Systems,” 2nd ESA Workshop on Advanced Flexible Telecom Payloads, 17-19 April 2012

${ }^{7}$ Gabellini P., Gatti N., Gallinaro G., Vecchi G., Della Croce F., Rinaldo R., Angeletti P., "Proposed Architecture of a System and Antenna/Payload Co-Design Tool for Multi-Beam Broadband Satellite Systems”, ESA Workshop on Advanced Flexible Telecom Payloads, 18-20 Nov. 2008.

${ }^{8}$ Alberti X., Cebrian, J. M., Del Bianco A., Katona Z., Lei J., Vasquez-Castro M. A., Zanus A., Gilbert L., Alagha N., "System Capacity Optimization in Time and Frequency for Multibeam Multi-media Satellite Systems", 5 ${ }^{\text {th }}$ Advanced Satellite Multimedia Systems Conference, Sep. 2010. 